\title{
Evolution Des Indices Pluviométriques Extrêmes Par L'analyse De Modèles Climatiques Régionaux Du Programme CORDEX: Les Projections Climatiques Sur Le Sénégal
}

\author{
Alioune Badara Sarr \\ Assane SECK university of Ziguinchor, Senegal \\ Moctar Camara \\ Laboratoire d'océanographie, des sciences de l'environnement et du climat \\ (LOSEC), UFR Sciences et Technologies - Université Assane SECK de \\ Ziguinchor, Senegal
}

doi: 10.19044/esj.2017.v13n17p206 URL:http://dx.doi.org/10.19044/esj.2017.v13n17p206

\begin{abstract}
This study aims at characterizing the extreme rainfall events over West Africa particularly in the Sahel region and Senegal by 2100 (far future) under the greenhouse gas emission scenario RCP8.5 by analyzing the simulations of four (4) regional climate models (RCMs) of CORDEX (Regional COordinated climate Downscaling Experiment) program. The study of these extreme climate indices is crucial for the understanding of the impacts of climate change on some vital socio-economic sectors such as the agriculture in Sahel and Senegal. The results show that almost all the RCMs predict a decrease of the rainfall over most parts of the Sahel region particularly over the Western Sahel. The analysis of the climate indices such as the highest one day precipitation amount, the 99th percentile and the maximum dry spell length (CDD) shows that the RCMs (except CanRCM4) project an increase of these exceptional rainfall events over the Sahel (especially over the Western Sahel) by 2100.

In Senegal, the RCMs (except RCA4) agree on a decrease of the precipitation and the number of wet days by 2100 . When considering the evolution of rainfall events intensity, the highest one day precipitation amount and the 99th percentile, the RCMs (except CanRCM4) predict an increase of the extreme events which may translate into strong floods in Senegal. As for the dry and wet sequences, the RCMs projections (except those of RCA4) show an increase (respectively a decrease) of the maximum dry spell length (respectively of the maximum wet spell length) in Senegal. This increase in extreme rainfall indices may translate into a strengthening of natural disasters such as floods and drought. This work can be considered as
\end{abstract}


a support for the policymakers in West Africa and particularly in Senegal for the better long-term planning of water resources and disaster management as wells as the build of a resilient agricultural system.

Keywords: Climate change, Regional Climate Models, CORDEX, climate indices, extreme rainfall events, West Africa, Senegal

\section{Résumé}

L'objectif de cette étude est d'utiliser la pluie simulée par 4 modèles climatiques régionaux (MCR) du programme CORDEX (COordinated Regional climate Downscaling Experiment) pour caractériser les événements extrêmes de pluie en Afrique de l'Ouest particulièrement au Sahel et au Sénégal pour le futur lointain (2100) sous le scénario d'émission de gaz à effet de serre RCP8.5. L'étude de ces événements pluviométriques extrêmes est cruciale pour la compréhension des effets des changements climatiques sur certains secteurs économiques clés telle que l'agriculture au Sahel et au Sénégal. Les résultats montrent que presque tous les MCR prédisent une diminution des précipitations sur une bonne partie du Sahel notamment sur sa partie Ouest. L'analyse des indices climatiques tels que le cumul maximal quotidien de précipitation, le $99^{\text {ème }}$ percentile des précipitations et la durée maximale des séquences sèches (CDD), montre que les MCR (excepté CanRCM4) prévoient une augmentation de ces événements pluvieux exceptionnels sur le Sahel, particulièrement sa partie Ouest à l'horizon 2100. Au Sénégal, les MCR s'accordent sur une diminution des précipitations et du nombre de jours pluvieux à l'horizon 2100 à l'exception du modèle RCA4. Concernant l'évolution des intensités de jours pluvieux, du cumul maximal quotidien et du $99^{\text {ème }}$ percentile des précipitations presque tous les MCR sauf CanRCM4 prévoient une augmentation des évènements extrêmes de pluie pouvant ainsi entrainer de fortes inondations sur le Sénégal. Pour ce qui est des séquences sèches et humides toutes les projections obtenues avec les MCR (à l'exception du modèle RCA4) montrent une augmentation (respectivement une diminution) de la durée maximale des séquences sèches (respectivement de la durée maximale des séquences humides) sur l'ensemble du Sénégal. Cette hausse des événements de précipitation extrêmes pourrait se traduire par une recrudescence des catastrophes naturelles telles que les inondations et la sécheresse qui pourraient influer négativement sur les activités socio-économiques des populations locales. Ce travail peut donc être considéré comme une aide à la décision pour les décideurs politiques dans l'élaboration des politiques publiques futures en matière de gestion des ressources en eau, de la sécurité alimentaire (agriculture résilient) et des catastrophes naturelles. 
Mots clés : Changement climatique, Modèles Climatiques Régionaux, CORDEX, Indices climatiques, Evénements extrêmes de précipitation, Afrique de l’Ouest, Sénégal

\section{Introduction}

L'Afrique de l'Ouest est une région où les populations sont toujours confrontées à une forte variabilité climatique. En plus de cette variabilité, les changements climatiques résultant des émissions anthropiques de gaz à effet de serre viennent s'ajouter à ces conditions difficiles. Beaucoup de projections climatiques ont montré que l'Afrique de l'Ouest va connaître une hausse importante des températures associée à une forte variabilité des précipitations (Kouame et al., 2013; Kouakou et al. 2014; Ly et al. 2013). Ces changements climatiques pourraient entrainer des conséquences néfastes sur l'environnement telles que les inondations ou la sécheresse (Giorgi et al. 2014, Sylla et al. 2016). L'agriculture qui est une source de revenue très importante pour cette région pourrait probablement être compromise par ce changement et ces variabilités climatiques (Boko et al. 2007, Ramin et Mc Michael 2009). C'est pourquoi depuis quelques décennies, les études d'impact du climat ne cessent de s'accroître. Pour y arriver plusieurs scénarios de changements climatiques ont été utilisés. Ces scénarios ont été souvent faits avec des modèles climatiques globaux (MCG). Cependant ces MCG rencontrent d'énormes problèmes pour représenter le climat dans cette région à cause de leur résolution spatiale très grossière ( 200 à $300 \mathrm{~km}$ ). Donc ces modèles sont importants quand il s'agit de représenter le climat sur une très grande échelle d'espace. Mais à l'échelle régionale, ils présentent de nombreuses limites (Hulme et al. 2001, Giannini et al. 2008). Pour remédier à ces difficultés on fait recours maintenant aux modèles climatiques régionaux (MCR) qui ont une résolution spatiale beaucoup plus fine $(\sim 10$ à $100 \mathrm{~km}$ ) (Camara et al. 2013). Dans cette étude, la pluie simulée par 4 MCR issus du programme CORDEX (COordinated Regional climate Downscaling EXperiment) est analysée afin de comprendre l'évolution de la pluie moyenne et des événements de précipitations extrêmes à l'horizon 2100 en Afrique de l'Ouest et particulièrement au Sénégal.

CORDEX est un programme international mis en œuvre par plusieurs centres de recherches dans le but de produire des scénarios de changement climatique fiables à des fins d'études d'impact. Ce projet met ainsi à la disposition de la communauté scientifique des scénarios haute résolution de changement climatique à l'échelle régionale sur la majeure partie des terres émergées de la planète. L’objectif de notre étude est d'étudier l'évolution du climat Ouest africain pour le futur lointain (2071-2100) avec un zoom sur le Sénégal en utilisant le scénario RCP8.5. Les données et méthodes utilisées 
sont présentées dans la seconde section. Dans la troisième section, les résultats sont exposés suivis de discussions dans la quatrième section.

\section{Données et méthodes:}

Des simulations haute résolution $\left(0.44^{\circ}\right)$ issues de $4 \mathrm{MCR}$ du programme CORDEX sont utilisées dans cette étude. Ces MCR sont présentés dans le tableau 1. Les MCR RACMO22T et CanRCM4 sont forcés respectivement par les MCG EC-EARTH et CanESM2; alors que les modèles RCA4 et CCLM4 sont forcés par les MCG CNRM-CM5. Au moment d'entamer cette étude, seules les sorties de ces quatre (4) modèles étaient disponibles sur la période d'étude envisagée. Les simulations des modèles climatiques régionaux du programme CORDEX peuvent être téléchargées sur le site: https://www.cordex.org/output.html

Deux périodes ont été considérées dans cette étude: la période de référence (1976-2005) et le futur lointain (2071-2100). Les modèles sont forcés pour le futur par le scenario RCP8.5 (Moss et al. 2010). Ce scénario correspond à une émission de $8.5 \mathrm{~W} / \mathrm{m} 2$ à l'horizon 2100. La distribution spatiale de la pluie durant l'été (Juillet-Septembre) est analysée de même que certains indices climatiques recommandés par l'organisation météorologique mondiale (OMM). Ces indices climatiques basés sur des données journalières de précipitation permettent de caractériser les événements pluvieux en Afrique de l'Ouest et particulièrement au Sénégal. Dans cette étude, sept (7) indices climatiques ont été considérés car pouvant potentiellement impacter sur la disponibilité des ressources en eau et la productivité agricole dans notre zone. Ces indices sont présentés dans le tableau 2 (Peterson et al, 2001). Les projections ont été réalisées sur l'Afrique. Dans ce travail, nous avons considéré l'Afrique de l'Ouest avec un zoom sur le Sénégal.

Les résultats sont présentés sous forme d'écart relatif : $I=100 \times \frac{\text { FL-HIST }}{\text { HIST }}$, où FL et HIST représentent respectivement la moyenne estivale sur le futur lointain (2071-2100) et la période historique (19762005).

Tableau 1: Description des modèles climatiques régionaux utilisés

\begin{tabular}{|c|c|c|}
\hline Nom & Institution & Références \\
\hline CCLM4 & $\begin{array}{c}\text { Climate limited-area Modelling } \\
\text { community }\end{array}$ & Baldauf et al. 2011 \\
\hline RACMO22T & $\begin{array}{c}\text { Royal Netherlands Meteorogical Institute, De Bilt, } \\
\text { The Netherlands }\end{array}$ & Meijgard et al. 2008 \\
\hline RCA4 & $\begin{array}{c}\text { Swedish Meteorological and Hydrological } \\
\text { Institute, Rossby Centre }\end{array}$ & $\begin{array}{c}\text { Samuelsson et al. } \\
2011\end{array}$ \\
\hline CanRCM4 & $\begin{array}{c}\text { CCCma (Canadien Centre for Climate Modelling } \\
\text { and analysis, Victoria, BC, Canada }\end{array}$ & \begin{tabular}{c} 
Scinocca et al. 2008 \\
\hline
\end{tabular} \\
\hline
\end{tabular}


Tableau 2 : Description des indices climatiques

\begin{tabular}{|c|c|}
\hline Indices & Signification \\
\hline Nombre de jours pluvieux & $\begin{array}{c}\text { Nombre de jours présentant un cumul pluviométrique } \\
\text { journalier supérieur à } 1 \mathrm{~mm}\end{array}$ \\
\hline Intensité des jours pluvieux & $\begin{array}{c}\text { Intensité moyenne de précipitation enregistrée pendant un } \\
\text { jour pluvieux }\end{array}$ \\
\hline Nombre de jours de pluies très intenses & $\begin{array}{l}\text { Nombre de jours pluvieux présentant un cumul } \\
\text { pluviométrique journalier supérieur à } 20 \mathrm{~mm}\end{array}$ \\
\hline Durée maximale des séquences humides & $\begin{array}{l}\text { Nombre maximal de jours consécutifs présentant un } \\
\text { cumul pluviométrique journalier supérieur à } 1 \mathrm{~mm}\end{array}$ \\
\hline Durée maximale des séquences sèches & $\begin{array}{l}\text { Nombre maximal de jours consécutifs présentant un } \\
\text { cumul pluviométrique journalier inférieur à } 1 \mathrm{~mm}\end{array}$ \\
\hline $\begin{array}{c}\text { Maximum des cumuls de précipitations } \\
\text { sur un jour }\end{array}$ & Le plus grand cumul pluviométrique enregistré sur un jour \\
\hline $99^{\text {ème }}$ percentile & $\begin{array}{l}\text { La valeur en dessous de laquelle se trouve 99\% des } \\
\text { données }\end{array}$ \\
\hline
\end{tabular}

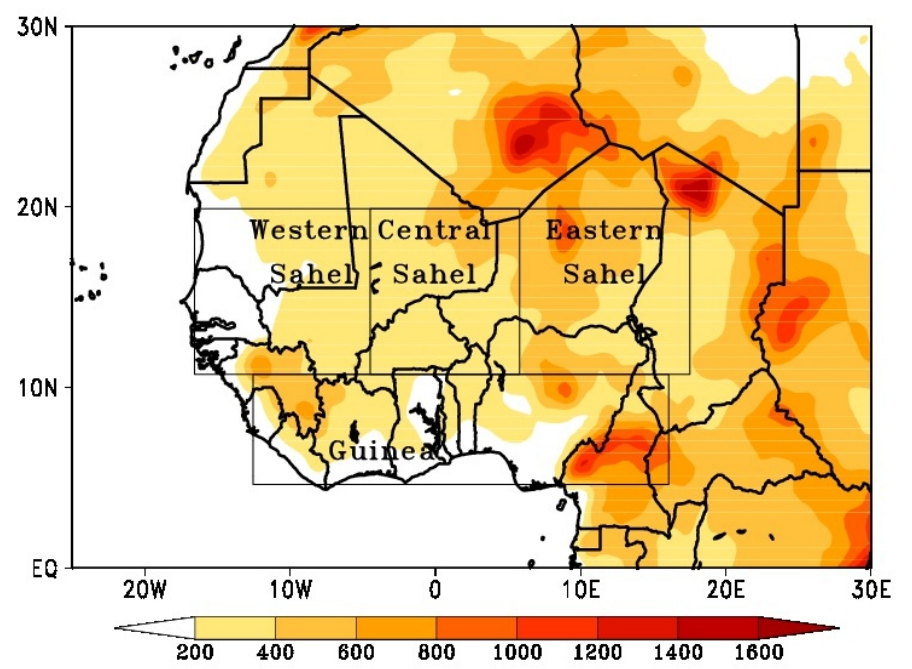

Figure 1 : Domaine d'étude (Afrique de l’Ouest) avec les parties considérées (Sahel Ouest, Sahel Central, Sahel Est et zone guinéenne)

\section{Résultats}

\section{Variabilité intra-saisonnière}

Les figures 2 et 3 présentent respectivement la pluie moyenne en été (juillet-septembre) sur l'Afrique de l'Ouest et sur le Sénégal.. Des différences importantes sont observées sur l'évolution des précipitations en Afrique de l'Ouest. Le modèle CCLM4 (figure 2a) prévoit un déficit de précipitation sur presque toute l'Afrique de l'Ouest exceptée la partie Ouest de la zone guinéenne. Le modèle CanRCM4 (figure 2d) prévoit une diminution de la pluie sur tout le Sahel Ouest et au Sud de $5^{\circ} \mathrm{N}$. Quant à RCA4, une faible diminution de la pluie est notée sur une bonne partie de l'Afrique de l'ouest. Quand nous considérons le modèle RACMO22T et la moyenne des modèles (figure $2 \mathrm{c}$ et $2 \mathrm{e}$ respectivement), ils prévoient une 
diminution du cumul pluviométrique sur le Sahel Ouest. Au Sénégal (figure 3), les MCR y prévoient généralement une forte diminution des hauteurs de pluie. Cependant, le modèle CCLM4 (figure 3a) prévoit une légère augmentation sur la partie sud-est du Sénégal (vers la région de Kédougou). Le modèle RACMO22T (figure 3c) prévoit aussi une faible augmentation au sud du pays, notamment entre les longitudes $15.5^{\circ} \mathrm{W}$ et $13^{\circ} \mathrm{W}$ (vers le sud de la région de Sédhiou). Les changements projetés sur le futur lointain du nombre de jours pluvieux ( $\mathrm{R}>1 \mathrm{~mm} / \mathrm{j}$, figure 4 et 5 ) montrent que le modèle CCLM4 (figure 4a) présente une structure dipolaire sur l'Afrique de l'Ouest qui se manifeste par une diminution du nombre de jours pluvieux sur le Sahel et une augmentation sur la zone guinéenne. Le modèle RACMO22T (figure 4c) prévoit une diminution sur tout le Sahel et un faible changement de nombre de jours de pluie sur la zone guinéenne comparé à la période de référence. CanRCM4 (figure 4d) de même que la moyenne des modèles (figure 4e) prévoient une diminution du nombre de jours pluvieux sur le Sahel Ouest et cela est cohérent avec l'évolution de la pluie moyenne. Les MCR prévoient une diminution du nombre de jours de pluie au Sénégal (figure 5) excepté le modèle RCA4 (figure 5b). Ce modèle prévoit un faible changement de nombre de jours de pluie entre le futur lointain et la période de référence sur presque tout le pays sauf sur la partie nord-ouest où il montre une légère augmentation de nombre de jours de pluie.

Quand on considère les intensités de jours pluvieux (figure non montrée) en Afrique de l'Ouest et au Sénégal, les MCR prévoient une augmentation sur toute l'Afrique de l'ouest en particulier sur le Sénégal excepté le modèle CanRCM4 qui prévoit une diminution sur tout le Sahel Ouest. 

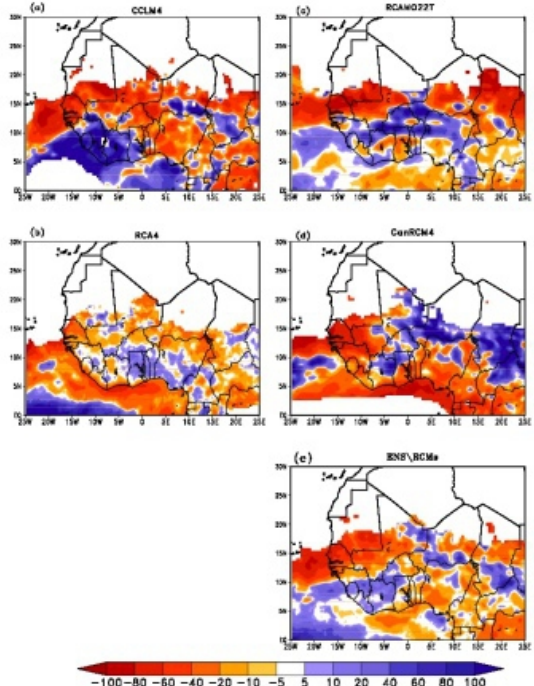

Figure 2: Ecart relatif de la pluie moyenne entre le futur lointain (2071) et la période historique (1976-2005) durant l'été (juillet-septembre) pour les modèles climatiques régionaux et leur moyenne forcés par le scénario de gaz à effet de serre RCP8.5 en Afrique de l'Ouest.
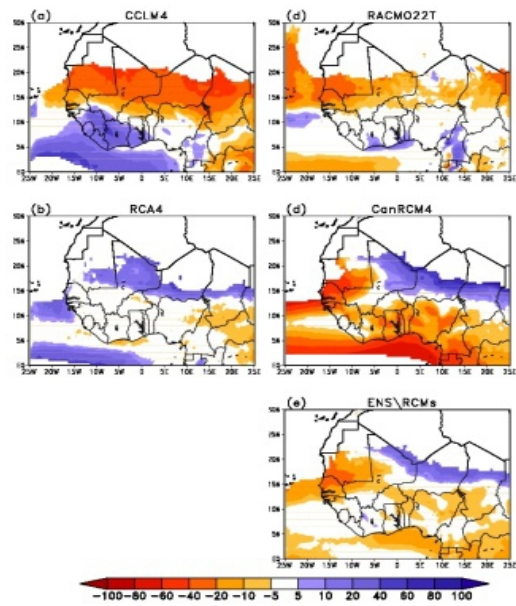

Figure 4: Ecart relatif du nombre de jours pluie ( $\mathrm{R}>1 \mathrm{~mm} / \mathrm{j}$ ) entre le futur lointain (2071-2100) et la période historique (1976-2005) durant l'été (juillet-septembre) pour les 4 modèles climatiques régionaux et leur moyenne forcés par le scénario de gaz à effet de serre RCP8.5 en Afrique de

l'Ouest.
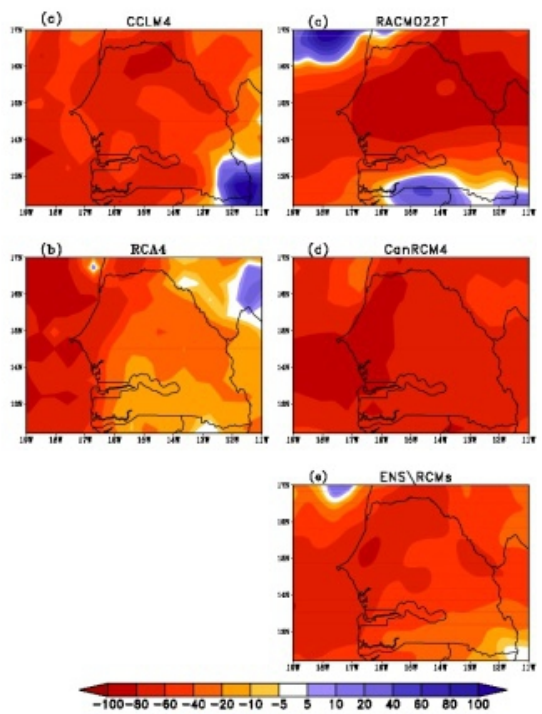

Figure 3: Ecart relatif de la pluie moyenne entre le futur lointain (2071) et la période historique (1976-2005) durant l'été (juillet-septembre) pour les modèles climatiques régionaux et leur moyenne forcés par le scénario de gaz à effet de serre RCP8.5 au Sénégal.
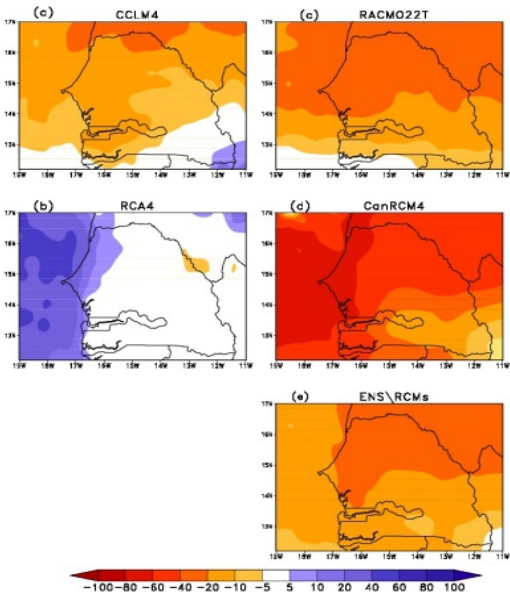

Figure 5: Ecarts relatif du nombre de jours pluie ( $R>1 \mathrm{~mm} / \mathrm{j}$ ) entre le futur lointain (2071-2100) et la période historique (1976-2005) durant l'été (juillet-septembre) pour les 4 modèles climatiques régionaux et leur moyenne forcés par le scénario de gaz à effet de serre RCP8.5 au Sénégal. 


\section{Variabilité des événements pluvieux extrêmes}

Pour caractériser les événements extrêmes pluvieux, nous avons analysé les indices climatiques suivants: le nombre de jours de pluies très intenses $(\mathrm{R}>20 \mathrm{~mm} / \mathrm{j})$, le $99^{\text {ème }}$ percentile des précipitations, le cumul maximal quotidien des précipitations quotidiennes (Rx1day), la durée maximale du nombre de jours consécutifs pluvieux (CWD) et la durée maximale du nombre de jours consécutifs secs (CDD).

Les écarts relatifs du nombre de jours de fortes pluies $(\mathrm{R}>20 \mathrm{~mm} / \mathrm{j})$ entre le futur lointain et la période historique sont représentés sur les figures 6 et 7, respectivement pour l'Afrique de l'Ouest et pour le Sénégal. Les projections du modèle CCLM4 (figure 6a) montrent une diminution du nombre de jours de fortes pluies sur le nord du Sahel Ouest, sur le Sahel Central et sur le Sahel Est. Le modèle RACMO22T (figure 6c) prévoit une diminution sur le Sahel Ouest et sur le Sahel Est. Sur le Sahel Central et sur la zone guinéenne, il y prévoit une augmentation des jours de fortes pluies. Les prévisions obtenues avec le modèle RCA4 (figure 6b) montrent une augmentation très importante des jours de fortes de pluies alors que le modèle CanRCM4 (figure 6d) prévoit une forte diminution sur le Sahel Ouest associée à une forte augmentation sur le reste du domaine. Quant à la moyenne des modèles (figure 6e), elle prévoit une diminution sur le nord du Sahel Ouest et une faible augmentation sur le reste du domaine. Sur le Sénégal, le modèle CCLM4 (figure 7a) prévoit une diminution sur sa partie Ouest et une faible augmentation sur sa partie Est. Les modèles RACMO22T et la CanRCM4 (respectivement figures 7c et 7d) prévoient une diminution très importante des jours de fortes de pluies sur presque tout le Sénégal excepté la partie Sud. Quand on considère le modèle RCA4 (figure $7 \mathrm{~b}$ ), le nombre de jours de pluies très intenses connaitra une forte augmentation sur tout le territoire sénégalais. La moyenne des modèles (figure 7e) présente une variation faible avec une légère augmentation sur sa partie Sud Est et une diminution vers le Nord du Sénégal. Les évolutions à l'horizon 2100 du $99^{\text {ème }}$ percentile et du cumul maximal quotidien sur l'Afrique de l'Ouest(respectivement figures 8 et 10) sont similaires. En effet, les MCR prévoient une évolution qui sera généralement à la hausse presque sur toute l'Afrique de l'Ouest malgré le fait qu'ils prévoient une diminution de la pluie sur le Sahel Ouest. En revanche CanRCM4 (figure 8c) prévoit une diminution sur le tout Sahel Ouest. Cette augmentation de ces événements pluviométriques extrêmes concernera aussi le Sénégal (figure 9 pour le $99^{\text {ème }}$ percentile et figure 11 pour le cumul maximal quotidien) pour la plupart des modèles. Par contre le modèle RACMO22T (figure 9c) prévoit une diminution au centre et au nord du pays pour le $99^{\text {ème }}$ percentile alors que pour le cumul maximal quotidien, il simule une légère diminution au nord du 
pays. Les prédictions de RCA4 (figure 9b) montrent aussi une diminution du cumul maximal quotidien au sud-ouest du pays.

La dernière partie de ce travail est consacrée à l'analyse des séquences humides et sèches pour le futur lointain. Presque tous les MCR s'accordent sur une diminution de la durée maximale du nombre de jours consécutifs humides sur l'Afrique de l'Ouest à l'horizon 2100 (figure 12). Cependant le modèle CanRCM4 (figure 12d) prévoit une légère augmentation sur le Nord du Sahel Central. Cette baisse prévue de la durée maximale des séquences humides à l'horizon 2100 est beaucoup plus significative sur le Sahel Ouest où elle peut atteindre 30 à 60\% par rapport à la période de référence (1976-2005). Cette diminution du nombre jours consécutifs humides affectera aussi le Sénégal selon la plupart des MCR excepté RCA4 (figure 13b) qui y prévoit une faible augmentation sur sa partie Est. Les figures 14 et 15 montrent respectivement les écarts relatifs sur la durée maximale du nombre de jours consécutifs secs entre le futur lointain et la période historique en Afrique de l'Ouest (figure 14) et au Sénégal (figure 15). CCLM4, RACMO22T et CanRCM4 (respectivement figures 14a, 14c et 14d) prévoient une augmentation des séquences sèches sur le Sahel Ouest. Quand nous considérons le modèle RCA4 (figure 14b), il prévoit une diminution sur le centre et sur l'Est du Sahel. La moyenne des modèles (figure 14e) présente un dipôle sur le Sahel qui se manifeste par une augmentation de la longueur des séquences sèches sur le Sahel Ouest associée à une diminution sur le Sahel Est.

Au Sénégal, presque tous les modèles y prévoient une augmentation des séquences sèches. Cependant les projections de RCA4 (figure 15b) y montrent une faible diminution (inférieur à $40 \%$ ) au centre et au nord du Sénégal. Sur la partie sud du pays, les modèles RCA4 et CCLM4 (figure 15a) montrent que les changements de la durée maximale du nombre de jours consécutifs secs sont généralement faibles. 

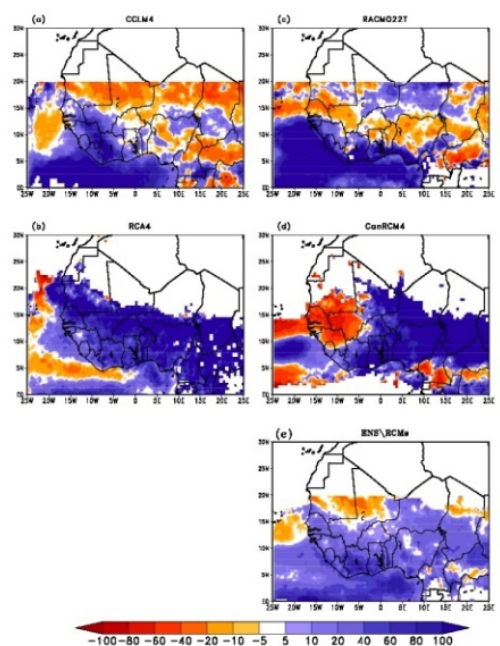

Figure 6: Ecarts relatif du nombre de jours de pluie très intenses $(\mathrm{R}>20 \mathrm{~mm} / \mathrm{j})$ entre le futur lointain (2071-2100) et la période historique (1976-2005) durant l'été (juillet-septembre) pour les 4 modèles climatiques régionaux et leur moyenne forcés par le scénario de gaz à effet de serre RCP8.5 en Afrique de l'Ouest.
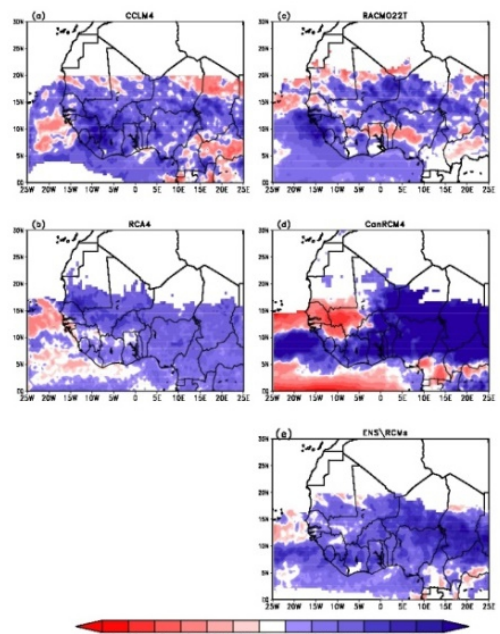

Figure 8: Ecart relatif du $99^{\text {ème }}$ percentile des précipitations entre le futur lointain (2071-2100) et la période historique (1976-2005) durant l'été (juillet-septembre) pour les 4 modèles climatiques régionaux et leur moyenne forcés par le scénario de gaz à effet de serre RCP8.5 en Afrique de l'Ouest.
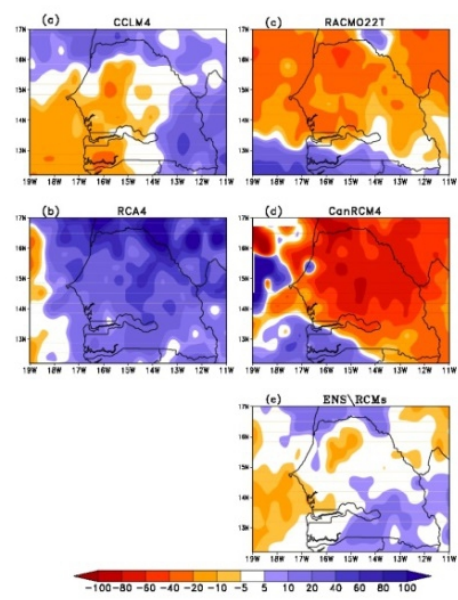

Figure 7: Ecarts relatif du nombre de jours de pluie très intenses $(\mathrm{R}>20 \mathrm{~mm} / \mathrm{j})$ entre le futur lointain (2071-2100) et la période historique (1976-2005) durant l'été (juillet-septembre) pour les 4 modèles climatiques régionaux et leur moyenne forcés par le scénario de gaz à effet de serre RCP8.5 au Sénégal.
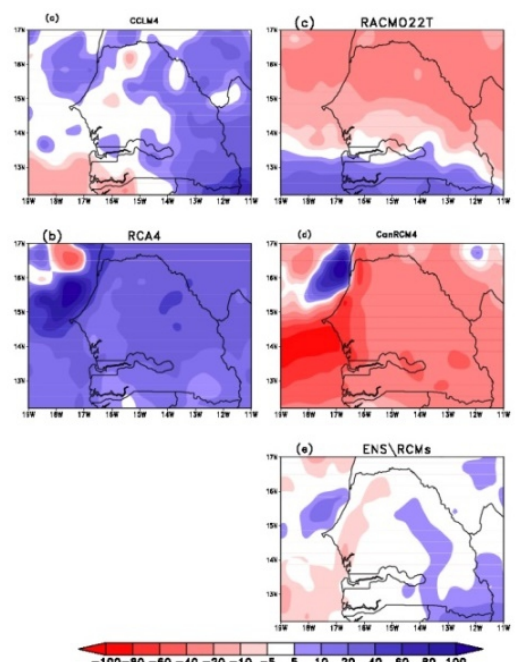

Figure 9: Ecart relatif du $99^{\text {ème }}$ percentile des précipitations entre le futur lointain (2071-2100) et la période historique (1976-2005) durant l'été (juillet-septembre) pour les 4 modèles climatiques régionaux et leur moyenne forcés par le scénario de gaz à effet de serre RCP8.5 au Sénégal. 

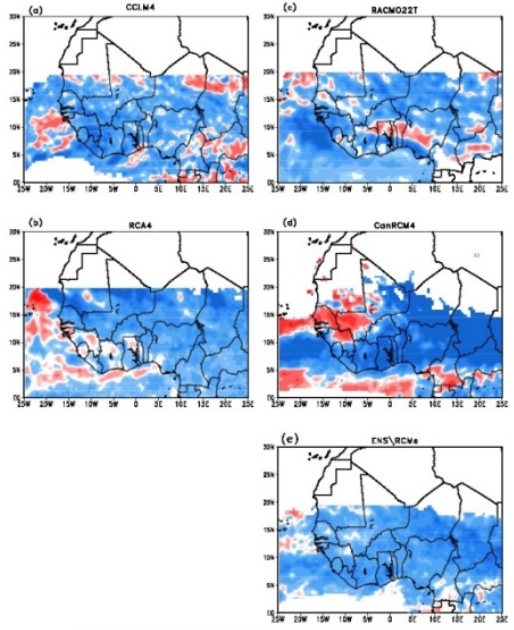

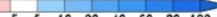

Figure 10: Ecarts relatif du cumul maximal quotidien des précipitations (Rx1day) entre le futur lointain (2071-2100) et la période historique (1976-2005) durant l'été (juillet-septembre) pour les 4 modèles climatiques régionaux et leur moyenne forcés par le scénario de gaz à effet de serre RCP8.5 en Afrique de l'Ouest.
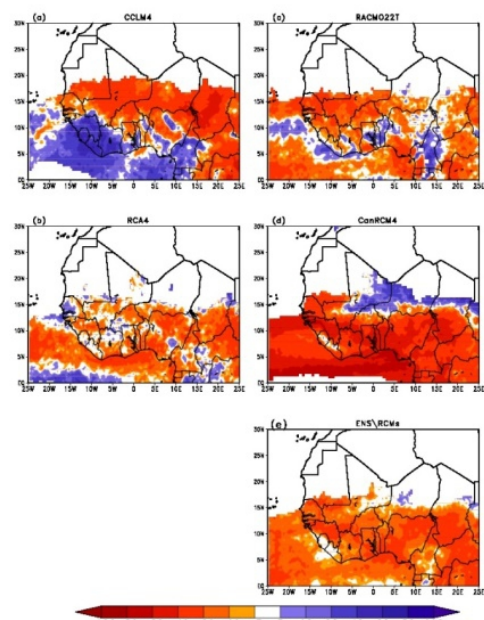

Figure 12: Ecart relatif de la durée maximale du nombre de jours consécutifs humides (CWD) entre le futur lointain (2071-2100) et la période historique (1976-2005) durant l'été (juilletseptembre) pour les 4 modèles climatiques régionaux et leur moyenne forcés par le scénario de gaz à effet de serre RCP8.5 en Afrique.
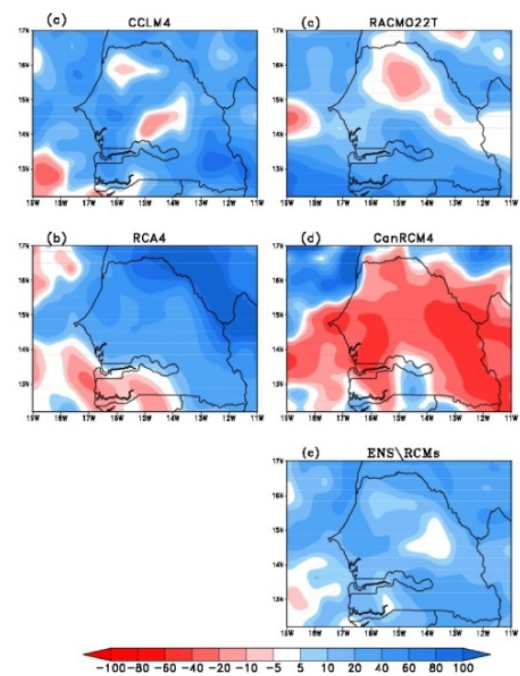

Figure 11: Ecart relatif du cumul maximal quotidien des précipitations (Rx1day) entre le futur lointain (2071-2100) et la période historique (19762005) durant l'été (juillet-septembre) pour les 4 modèles climatiques régionaux et leur moyenne forcés par le scénario de gaz à effet de serre RCP8.5 au Sénégal.
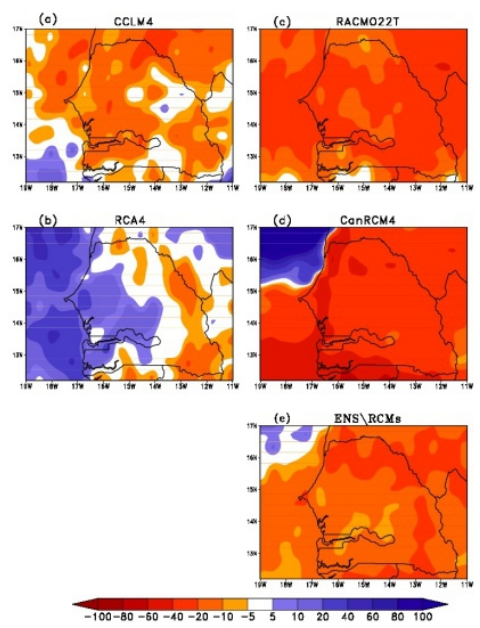

Figure 13: Ecart relatif de la durée maximale du nombre de jours consécutifs humides (CWD) entre le futur lointain (2071-2100) et la période historique (1976-2005) durant l'été (juilletseptembre) pour les 4 modèles climatiques régionaux et leur moyenne forcés par le scénario de gaz à effet de serre RCP8.5 au Sénégal. 


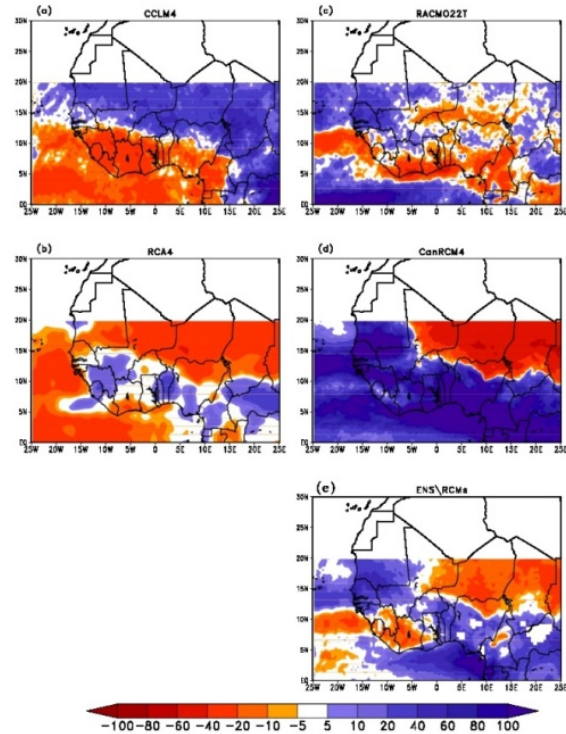

Figure 14: Ecart relatif de la durée maximale du nombre de jours consécutifs secs (CDD) entre le futur lointain (2071-2100) et la période historique (1976-2005) durant l'été (juilletseptembre) pour les 4 modèles climatiques régionaux et leur moyenne forcés par le scénario de gaz à effet de serre RCP8.5 en Afrique de l'Ouest.
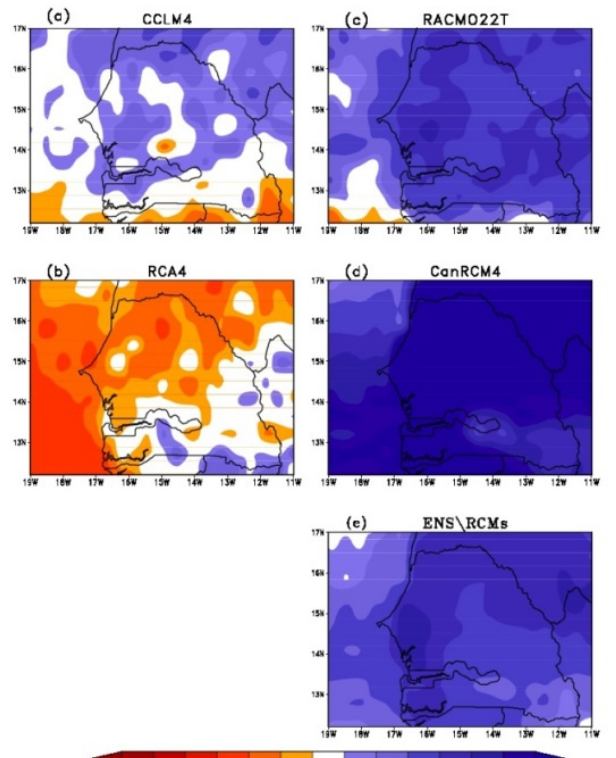

Figure 15: Ecart relatif de la durée maximale du nombre de jours consécutifs secs (CDD) entre le futur lointain (2071-2100) et la période historique (1976-2005) durant l'été (juillet-septembre) pour les 4 modèles climatiques régionaux et leur moyenne forcés par le scénario de gaz à effet de serre RCP8.5 au Sénégal.

\section{Discussions}

La forte disparité notée sur l'évolution des précipitations à l'horizon 2100 pourrait être due au type de forçage ou au schéma de convection utilisé sur l'Afrique de l'Ouest (Sylla et al. 2011; Klutse et al. 2015). Cette divergence des modèles montre aussi que les projections climatiques sur les précipitations en Afrique de l'Ouest sont encore incertaines (GIEC 2013; Sylla et al. 2016). Cependant, sur le Sahel Ouest les modèles analysés s'accordent sur une diminution des précipitations. Kouakou et al. (2014) ont estimé cette diminution des précipitations sur le Sahel Ouest à environ 12.6\% à l'horizon 2091-2100. Diallo et al. (2016) ont souligné que la diminution de la pluie moyenne dans cette région de l'Afrique de l'Ouest pourrait probablement être induite en partie par un affaiblissement de l'humidité provenant des sources locales, ce qui ralentit le cycle hydrologique. Sur le Sénégal, nos travaux sont cohérents avec ceux de Tall et al. (2016) qui ont trouvé une forte diminution des précipitations à l'horizon 2080-2099 sous le scénario RCP8.5. 
Quand on considère le $99^{\text {ème }}$ percentile et le cumul maximal quotidien, il apparait que leur évolution (hausse) est contradictoire avec celle de la pluie moyenne simulée sur le Sahel Ouest, particulièrement au Sénégal. Cette étude montre donc que l'augmentation des précipitations extrêmes ne correspond pas toujours à une hausse de la pluie moyenne. La hausse des pluies extrêmes pourrait entrainer une recrudescence de certains événements extrêmes telles que les inondations. Ces résultats obtenus sur le Sahel Ouest sont aussi en accord avec ceux de Giorgi et al. 2011, 2014, de Sylla et al. 2012 et de Diallo et al. 2016. L'analyse de la durée maximale des séquences humides montre une forte diminution à l'horizon 2100 sur l'Afrique de l’Ouest, particulièrement au Sahel et au Sénégal. Ly et al. (2013) ont montré une diminution progressive de la durée maximale du nombre des séquences humides sur le Sahel depuis 1960. Nos travaux montre qu'une telle tendance diagnostiquée sur le climat présent risque de persister dans le futur. Cette diminution de la durée maximale des séquences humides sur l'ensemble du Sahel Ouest et au Sénégal en particulier semble être cohérente avec l'évolution de la durée maximale des séquences sèches car une forte diminution de la durée maximale des séquences humides pourrait favoriser une augmentation de la longueur maximale des séquences sèches. Cette diminution (respectivement augmentation) des séquences humides (respectivement séquences sèches) sur le Sahel Ouest est une réponse au réchauffement climatique global (Mariotti et al. 2014 ; Sylla et al. 2016). Une telle situation au Sahel Ouest et particulièrement au Sénégal pourrait avoir des conséquences néfastes sur certaines activités humaines qui dépendent fortement de la pluie estivale tels que l'agriculture pluviale et l'élevage (Salack et al. 2012). Les résultats de cette étude pourrait permettre aux décideurs politiques de mettre en place les stratégies d'adaptation nécessaires pour une meilleure gestion des ressources en eau et des catastrophes naturelles ainsi que l'élaboration d'un système agricole résilient face aux changements climatiques.

\section{Conclusion}

Cette étude réalisée avec $4 \mathrm{MCR}$ du programme CORDEX a permis d'analyser l'évolution des précipitations (moyenne et événements extrêmes) sur l'Afrique de l'Ouest avec un focus sur le Sénégal. Les MCR ainsi que leur moyenne ont montré que la pluie va diminuer sur une bonne partie de l'Afrique de l'Ouest surtout sur le Sahel Ouest. Certains indices climatiques ont été aussi analysés pour pouvoir caractériser les événements pluvieux exceptionnels. Selon les MCR, le nombre de jours pluvieux diminuera sur le Sahel, notamment sur le Sénégal et augmentera sur la zone guinéenne. Pour ce qui est du nombre de jours de pluies très intenses $(\mathrm{R}>20 \mathrm{~mm} / \mathrm{j})$, les modèles présentent des structures très variables mais la 
moyenne des modèles montre une augmentation sur presque toute l'Afrique de l’Ouest exceptée sur la partie Nord-Ouest du Sahel. Au Sénégal, presque tous les modèles sauf RCA4 prévoient une diminution des jours de fortes pluies et cela pourrait entrainer une augmentation des pauses de pluie dans ce pays. Quand on considère les intensités de jours pluvieux, le $99^{\text {ème }}$ percentile des précipitations, le cumul maximal quotidien, ces indices ont tendance à augmenter sur une bonne partie de l'Afrique de l'Ouest excepté pour le modèle CanRCM4 qui prévoit une diminution sur le Sahel Ouest. Les projections des MCR montrent que ces événements pluvieux extrêmes n’évoluent pas dans le même sens que les précipitations; cela pourrait entrainer ainsi des inondations intenses dans certaines parties de l'Afrique occidentale telle que le Sahel Ouest. Au Sénégal, les indices de précipitations extrêmes présente une tendance à la hausse selon les modèles CCLM4 et RCA4. L'analyse des séquences humides et sèches montre que la plupart des MCR présentent une diminution assez importante de la durée maximale du nombre de jours consécutifs pluvieux presque sur toute l'Afrique de l'Ouest. L’analyse de la durée maximale du nombre de jours consécutifs secs montre que les MCR présentent des différences importantes mais la plupart des modèles prévoit une augmentation de celle-ci sur le Sahel Ouest, notamment sur le Sénégal; cela pourrait entrainer des sécheresses intenses aux conséquences catastrophiques pour l'économie locale.

Les résultats de cette étude pourront aider à augmenter la résilience face aux changements climatiques de certaines activités humaines telles que l'agriculture qui est une source de nourriture et de revenue très importante pour les populations de l'Afrique de l'Ouest. En définitif, il est nécessaire d'approfondir ce travail dans le but d'améliorer les études d'impact du changement climatique sur l'écosystème naturel et sur certains activités humaines telles que l'agriculture en Afrique de l'ouest; région connue pour sa faible capacité d'adaptation.

\section{References:}

1. Ali A (2011): Climate variability and change in the Sahel. Understanding the current

2. situation by observing Climate change in the Sahel. A challenge for sustainable development. AGRHYMET Monthly Bulletin (Special Issue), pp. 17-20.

3. Baldauf M, Seifert A, Förstner J, Majewski D, Raschendorfer M, Reinhardt T (2011): Operational convective-scale numerical weather prediction with the COSMO model: description and sensitivies. Mon Wea Rev. doi: 10.1175/MWR.-D-10-05013.1

4. Boko M, Niang I, Nyong A, Vogel C, Githeko A, Medany M, Osman-Elasha B, Tabo R, Yanda P (2007): Africa. Climate change : 
impact adaptation and vulnerability. In: parry M.L, Canziani O. F, Palutikof JP, van der Linden PJ, Hanson C.E (eds) Contribution of working group II to the fourth assessment report of the intergovernmental panel on climate change Cambridge University Press, Cambridge, pp 433-467.

5. Camara M, Diédhiou A, Sow BA, Diallo MD, Diatta S, Mbaye I, Diallo I (2013): Analyse de la pluie simulée par les modèles climatiques régionaux de CORDEX en Afrique de l'Ouest. Sécheresse, 2013, Volume 24, N¹.

6. Diallo I, Giorgi F, Deme A, Tall M, Mariotti L, and Gaye AT (2016): Projected changes of summer monsoon extremes and hydroclimatic regimes over West Africa for the twenty-first century," Climate Dynamics, 2016.

7. Kouakou KE, Kouadio ZA, Kouassi FW, Goula Bi TA, Savané I (2014) : Modélisation de la température et de la pluviométrie dans un contexte de changement climatique : cas de l'Afrique de l'Ouest. Afrique Sciences. 10(1) (2014) 145 - 160

8. GIEC, Résumé à l'intention des décideurs, Changements climatiques (2013): Les éléments scientifiques. Contribution du Groupe de travail I au cinquième Rapport d'évaluation du Groupe d'experts intergouvernemental sur l'évolution du climat [sous la direction de Stocker TF, Qin D, Plattner GK, Tignor M, Allen SK, Boschung J, Nauels A, Xia Y, Bex V et Midgley PM]. Cambridge University Press, Cambridge, Royaume-Uni et New York (État de New York), États-Unis d'Amérique.

9. Kouamé YM, Soro GE, Kouakou KE, Kouadio ZA, Meledje NEH et al (2013): Scénarios des changements climatiques pour les précipitations et les températures en Afrique subsaharienne tropicale humide : Cas du bassin versant de Davo, Côte d'Ivoire, Larhyss Journal, ISSN 1112-3680, n²18, Juin 2014, pp. 197-213.

10. L’Hote, Y., Mahé, G., Some, B., Triboulet, J.P (2002): Analysis of a Sahelian annual rainfall index from 1896 to 2000; the drought continues. Hydrological Sciences-Journal 47 (4), 563-572.

11. Ly M., Traoré S. B., Alhassane A., Sarr B (2013): Evolution of Some Observed Climate Extremes in the West African Sahel. Weather and Climate Extreme, 19-25, http://dx.doi.org/10.1016/j.

12. Moss R, Babiker M, Brinkman S, Calvo E et al. (2007): Towards new scenarios for analysis of emissions, climate change, impacts and response strategies. IPCC expert meeting report, Noordwijkerhout, The Netherlands.

13. Nikulin G, Jones C, Samuelson P, Giorgi F, Sylla MB, Asrar G, Büchner M, Cerezo-Mota R, Christensen OB, Déqué M, Fernandez 
M, Hänsler A, van Meijgaard E, Sushama L (2012): Precipitation climatology in an ensemble of CORDEX-Africa regional climate simulations. Journal of climate. doi 10.1174/JCLI-D-11-00375.1.

14. Moss R, Babiker M, Brinkman S, Calvo E et al (2007): Towards new scenarios for analysis of emissions, climate change, impacts and response strategies. IPCC expert meeting report, Noordwijkerhout, The Netherlands.

15. Nikulin G, Jones C, Samuelson P, Giorgi F, Sylla MB, Asrar G, Büchner M, Cerezo-Mota R, Christensen OB, Déqué M, Fernandez M, Hänsler A, van Meijgaard E, Sushama L (2012): Precipitation climatology in an ensemble of CORDEX-Africa regional climate simulations. Journal of climate. doi 10.1174/JCLI-D-11-00375.1.

16. Peterson TC, Folland C, Gruza W, Hogg G, Mokssit A and Plummer N (2001): "Report on the activities of the working group on climate change detection and related rapporteurs 1998-2001," WMO Rep.WCDMP 47,WMO-TD 1071,.

17. Ramin BM and McMichael AJ (2009): Climate change and health in sub-saharan Africa: a case-based perspective. EcoHealth 6, 52-57. Doi: 10.1007/s10393-009-0222-4.

18. Salack S, Muller B, Gaye AT, Hourdin F, Cisse N (2012) : Analyses multi-échelles des pauses pluviométriques au Niger et au Sénégal. Sécheresse 23; 3-13. doi: 10.1684/sec.2012.0335

19. Samuelsson P, Jones CG, Willen U, Ullerstig A, Gollvik S, Hansson U, et al. (2011): The Rossby centre regional climate model RCA3: model description and performance. Tellus A 63: 4-23.

20. Scinocca JF, McFarlane NA , Lazare M, Li J, and Plummer D (2008): Technical Note: The CCCma third generation AGCM and its extension into the middle atmosphere. Canadian Centre for Climate Modelling and Analysis, Environment Canada, Victoria, B. C., Canada, Atmos. Chem. Phys., 8, 7055-7074.

21. Sylla MB, Gaye AT, Jenkins G.S (2012): On the fine-scale topography regulating changes in atmospheric hydrological cycle and extreme rainfall over West Africa in a regional climate model projections. International Journal of Geophysics, 981649. doi:10.1155/2012/ 981649.

22. Sylla MB, Nikiema PM, Gibba P, Kebe I, Klutse NAB (2016): Climate Change over West Africa: Recent Trends and Future Projections. J.A. Yaro and J. Hesselberg (eds.), Adaptation to Climate Change and Variability in Rural West Africa, DOI 10.1007/978-3-319-31499-0_3

23. Tall M, Sylla MB, Diallo I, Pal JS, Faye A, Mbaye ML, Gaye AT (2016): Projected impact of climate change in the hydroclimatology 
of Senegal with a focus over the Lake of Guiers for the twenty-first century. Theor Appl Climatol. doi:10.1007/s00704-016-1805-y

24. van MeijgaardE, van Ulft L, van de Berg WJ, Bosveld FC, van den Hurk B, Lenderink G, and Siebesma AP (2008): The KNMI regional atmospheric climate model RACMO version 2.1, Tech. Rep. 302. 